\section{Factors influencing the delivery of cancer pathways: a summary of the literature}

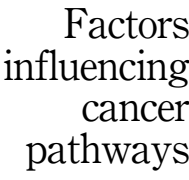

121

Syaribah Noor Brice and Paul Harper

School of Mathematics, Cardiff University, Cardiff, UK

Tom Crosby

Velindre Cancer Centre, Cardiff, UK

Daniel Gartner

School of Mathematics, Cardiff University, Cardiff, UK

Edilson Arruda

Department of Decision Analytics and Risk, Southampton Business School,

University of Southampton, Southampton, UK and

Alberto Luiz Coimbra Institute-Graduate School and Research in Engineering,

Federal University of Rio de Janeiro, Rio de Janeiro, Brazil

Tracey England

Department of Decision Analytics and Risk, Southampton Business School,

University of Southampton, Southampton, UK

Emma Aspland

School of Mathematics, Cardiff University, Cardiff, UK, and

Kieran Foley

Velindre Cancer Centre, Cardiff, UK
Received 21 May 2020 Revised 16 October 2020

24 December 2020

14 January 2021 Accepted 27 January 2021

\begin{abstract}
Purpose - The study aims to summarise the literature on cancer care pathways at the diagnostic and treatment phases. The objectives are to find factors influencing the delivery of cancer care pathways; to highlight any interrelating factors; to find gaps in the literature concerning areas of research; to summarise the strategies and recommendations implemented in the studies.

Design/methodology/approach - The study used a qualitative approach and developed a causal loop diagram to summarise the current literature on cancer care pathways, from screening and diagnosis to
\end{abstract}

(c) Syaribah Noor Brice, Paul Harper, Tom Crosby, Daniel Gartner, Edilson Arruda, Tracey England, Emma Aspland and Kieran Foley. Published by Emerald Publishing Limited. This article is published under the Creative Commons Attribution (CC BY 4.0) licence. Anyone may reproduce, distribute, translate and create derivative works of this article (for both commercial and non-commercial purposes), subject to full attribution to the original publication and authors. The full terms of this licence may be seen at http://creativecommons.org/licences/by/4.0/legalcode

This work has resulted from research funded by a Cancer Research UK grant Analysis and Modelling of a Single Cancer Pathway Diagnostics (Early Diagnosis Project Award A27882).

The authors would like to take this opportunity to thank all those staff in Velindre Cancer Centre, Aneurin Bevan University Health Board, Cwm Taf Morgannwg University Health Board, Welsh Government, Public Health Wales, NHS Wales and Cancer Research UK, who have supported this work through their valuable advice. In particular the authors would like to thank Karen Fitzgerald, Programme Director - ACE, at Cancer Research UK for her detailed and helpful feedback that has helped shape this paper.

Conflict of interest: None. 
JHOM

35,9

122 treatment. A total of 46 papers was finally included in the analysis, which highlights the recurring themes in the literature.

Findings - The study highlights the myriad areas of research applied to cancer care pathways. Factors influencing the delivery of cancer care pathways were classified into different albeit interrelated themes. These include access barriers to care, hospital emergency admissions, fast track diagnostics, delay in diagnosis, waiting time to treatment and strategies to increase system efficiency.

Originality/value - As far as the authors know, this is the first study to present a visual representation of the complex relationship between factors influencing the delivery of cancer care pathways.

Keywords Qualitative mapping, Cancer care pathways, Diagnostics, Treatment, Access barriers,

Literature review

Paper type Literature review

\section{Introduction}

A care pathway can be defined as a general clinical plan that maps the trajectory of the patient through the healthcare system (Altini et al., 2020; Schrijvers et al., 2012). As such, it involves many activities and can be seen as a complex adaptive system that responds to medical decisions, patient outcomes and local characteristics, among other factors. Indeed, care pathways are subjected to a complex interplay of factors that can be captured, for example using causal loop diagrams (Littlejohns et al., 2018; Sterman, 2000).

The importance of proper management and a clear understanding of the impact of care pathways in the quality of care is acknowledged in the literature (Allen et al., 2019). It is often argued that the input of patients, as well as healthcare specialists, is essential in the design of effective care pathways (Donetto et al., 2019). Whilst the design of care pathways to provide better patient experience and improved outcomes is an important area in the literature (Currie and Harvey, 2000; Lawson et al., 2006), this paper focuses on the analysis of the delivery of cancer care pathways to understand the complex interaction of factors that ultimately affect the quality of the service delivered to the patient.

Cancer is regarded as the second leading cause of death worldwide and accounts for approximately one in every six deaths around the globe (World Health Organization, 2018). It is estimated that half of the UK population will develop cancer in their lifetime (Queen Mary University of London, 2017).

Problems related to the availability and delivery of cancer care are common (Prager et al, 2018). The issues include the accessibility of cancer care services such as screening, diagnosis, treatment and the unpredictability in the delivery of these services (Wang and Onega, 2015). Several countries have developed standardised pathways for cancer patients to address the latest issue and improve patient outcomes. Descriptions of standardised lung cancer pathways in Australia, the UK and Canada can be found in Department of Health and Human Services (2016); Lung Clinical Expert Group (2017); Cancer Care Ontario (2019), respectively. Elsewhere, standardised pathways for cancer care have been found to correlate with improved survival rates for patients with seven distinct types of cancer in Denmark (Jensen et al., 2017). The pathways also contributed to the reduction of the diagnostic interval, i.e. the time elapsed from initial presentation to final diagnosis (Weller et al., 2012). Some studies have associated the implementation of standardised cancer pathways with increased efficiency, improved outcomes and higher patient satisfaction levels (e.g. Delilovic et al., 2019; Gesme and Wiseman, 2011). However, negative effects have also been identified, such as longer waiting times for patients competing for the same resources (e.g. Delilovic et al, 2019). This suggests that research on the implementation of standardised pathways that use shared resources should also consider the different patient groups sharing the resources.

Patient profiles may vary, and even the experiences of similar patients may be significantly different. Generally, variations may be due to the underlying heterogeneity of a patient's physical health and behaviours, professional uncertainty, external constraints, or 
diffusion of new knowledge and practices (Alzahouri et al., 2008). For example, similar patients may undertake distinct diagnostic procedures (Alzahouri et al., 2008); or the indications and the choice of emergency surgery procedures may vary according to local conditions (Bosscher et al., 2015). These uncertainties may further complicate an already involved decision-making process, but one way to mitigate their influence is by designing tools and patient pathways that benefit from multidisciplinary team discussions (Bosscher et al., 2015).

A report from the Organisation for European Cancer Institutes Accreditation and Designation program suggested that different cancer centres tend to have different numbers of pathologies with dedicated clinical pathways (Saghatchian et al., 2014). The variation also exists in the implementation of pathways with waiting time targets. In the UK, the National Health Service (NHS) guidelines established maximum waiting times of 2 weeks from referral to outpatient appointment and 62 days from referral to first treatment (Department of Health, 2007).

Literature review studies have explored factors relating to cancer care delivery, albeit with different focuses. The effect of case management in cancer care could not be ascertained due to the scarcity of literature and variations in methodology (Wulff et al., 2008). On the other hand, delays due to both the practitioner and the patient have been correlated with similar risk factors, such as demographic, socio-economic, education and health conditions (Macleod et al., 2009). In addition, the lack of knowledge about cancer symptoms and the benefits of treatment were found to influence the delays in accessing the service (Akuoko et al., 2017; Jones et al., 2014; Williams et al., 2019).

The aforementioned literature studies have investigated factors related to either delay in the presentation or diagnosis and treatment. In contrast, this study aims to summarise the literature on the delivery of cancer care pathways, covering the studies from presentation to diagnosis and treatment. The objectives include investigating the factors associated with the timely and effective delivery of the cancer pathways, highlighting any interrelating factors, summarising the implemented strategies for delivering cancer pathways and reporting gaps in the literature.

\section{Methodology}

The literature search was conducted in March 2020 and covered SCOPUS, Science Direct, MEDLINE, PubMed, Web of Science, and PMC. The search used the following keywords: cancer* AND ("diagnostic pathway*" OR "patient pathway*" OR "care pathway*" OR "critical pathway*" OR "care map*" OR "clinical pathway*"). We constrained ourselves to studies written in English in the last 20 years. We excluded studies that covered topics related to testing devices, development of tools, genetic testing, tumour growth and surgical procedures. The quality of the papers was not considered. The selection process was administered by the lead author. The result was presented, discussed and disseminated to the team members who gave suggestions and contributed to the writing of the paper.

Figure 1 summarises the selection process. The first filter identified 1,969 potential studies. This number reduced to 296 following the removal of duplicates and screening based on titles and abstracts. It was further trimmed to 151 after excluding content that did not comply with the inclusion criteria. Finally, 105 studies were excluded due to reasons such as being related to palliative or supportive care and not concerning the delivery of cancer pathways. This resulted in the selection of 46 papers for further analysis.

\subsection{Causal loop diagram for cancer care pathways}

Causal loop diagrams (Sterman, 2000) are a system's dynamics tool developed to convey the interplay of factors in complex social systems by promoting a holistic view of the problem 


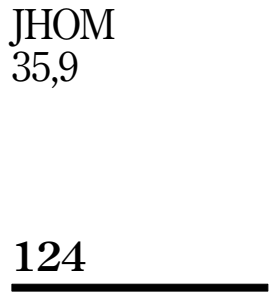

Figure 1.

The selection process flow using simplified PRISMA diagram

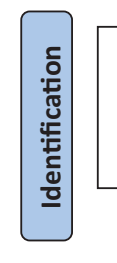

$$
\begin{aligned}
& \text { Records identified through } \\
& \text { database searching (Scopus) } \\
& (\text { ( } n=1875)
\end{aligned}
$$

Additional records identified

through other sources (Science

Direct, MEDLINE/PubMed,

Web of Science, PMC $(n=94)$
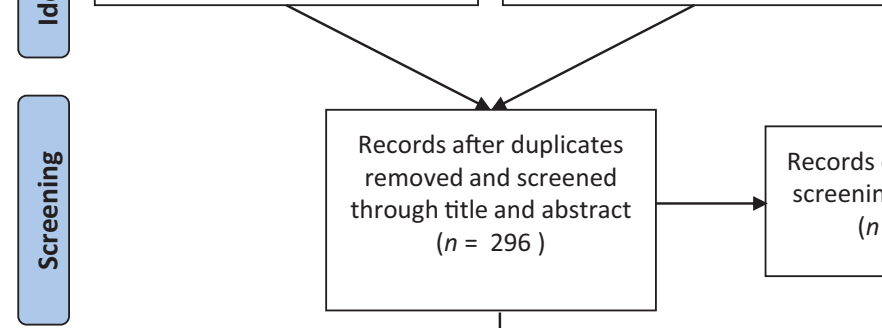
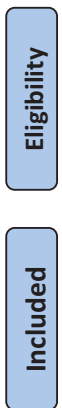

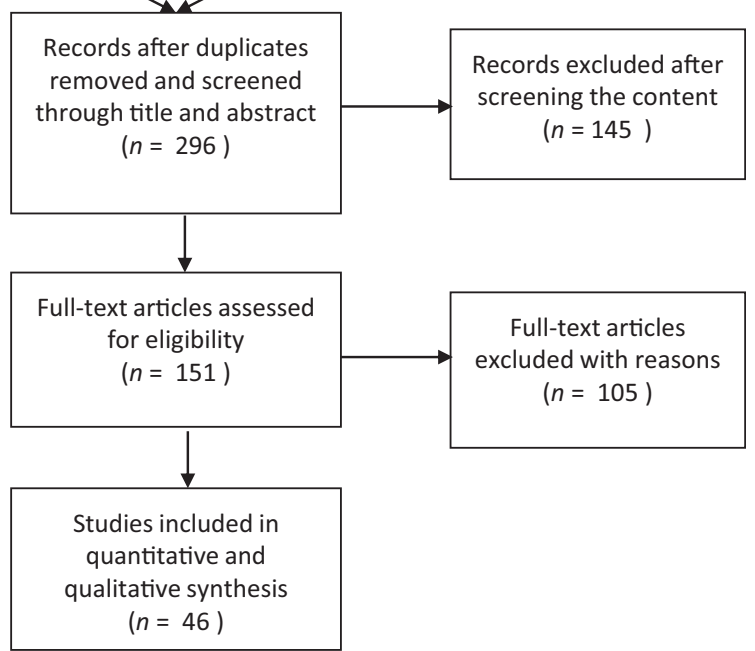

at hand. The rationale is to develop a diagram that conveys the existing relationships between pairs of factors to unveil a complete picture of the system to stakeholders and decision-makers.

The present study develops a causal loop diagram to highlight the causal link or relationship between each pair of factors/topics (also dubbed variables) investigated in the studies. In order to simplify the analysis whilst also retaining the essence of the articles, we make use of the topics that appear more frequently across all studies. The links between pairs of factors (variables) are represented by arrows in the resulting diagram. The arrows may be non-directional, when the relationship exists but no causality is attributed. Another type of arrow is uni-directional when the direction of causality is established. This type of arrow will have polarity (i.e. an established direction). The diagram does not capture any bi-directional relationship, since such a type of relationship was not found in the studies. It is important to note that the use of the tool is to facilitate the description of relationships between factors. These may not necessarily represent causality.

The factors were selected from the surveyed studies, as well as their pairwise relationships. For the sake of illustration, consider for example a study by Hansen et al. (2008) that investigated factors related to delay in cancer diagnosis. The study found that patients' socio-economic situation and age correlated with the delay in cancer diagnosis. Based on these results, the socio-economic and age factors were linked to patient delay in the diagram, see Figure 9.

The causal loop diagram was built using the software Vensim PLE, version 7.3.5 from Ventana Systems Inc (https://vensim.com). Individual analyses of specific themes were also carried out to describe pertinent variables following the surveyed literature. 


\section{Results}

Figure 2 highlights the number of works published yearly from 2008 to 2020. It displays an upward tendency in the number of yearly publications from 2012 to 2018 , which might indicate an increasing interest in the subject. Interestingly, no publications were found in 2009 and 2010, which is not to say that no research was conducted in that period. Overall, quantitative research was more prevalent than qualitative research.

Figure 3 presents the classification of the literature based on the types of services and cancers found in the literature. The number of papers dealing with "Diagnostics" stands out $(n=27)$, followed by studies concerned with "Treatment" and "Screening" ( $n=7$ and $n=6$, respectively). The category "Other" included studies related to efficiency and emergency admissions $(n=4)$. Studies on diagnostics mainly involved multiple types of cancer. We identified five emerging and important themes related to cancer care services, namely hospital emergency admissions, fast track diagnostics, efficient delivery of cancer care services, delay in diagnosis and waiting time to treatment, and barriers to care. Figure 4 shows the distribution of these themes according to the cancer specialties. Delay in diagnosis and waiting time to treatment is the most common theme in the surveyed studies $(n=15)$, followed by efficient delivery of care services $(n=10)$. The next most frequent theme is fast track diagnostics $(n=8)$, followed by access barriers to care $(n=7)$. Studies on fast track diagnostics mainly covered multiple cancer types $(n=5)$. Finally, the majority of lung cancer studies explored issues related to delay in diagnosis and waiting time to treatment $(n=5)$.

The derived causal loop diagrams feature emerging themes. Further analyses describe the relations between factors in the form of causal trees. Each subsequent subsection conveys a causes tree related to a specific theme and further discusses the theme. The theme delayed diagnosis and waiting time to treatment is further split into delay in diagnosis, and treatment delay. Given the complexity of the problem, each individual causes tree may fail to capture the
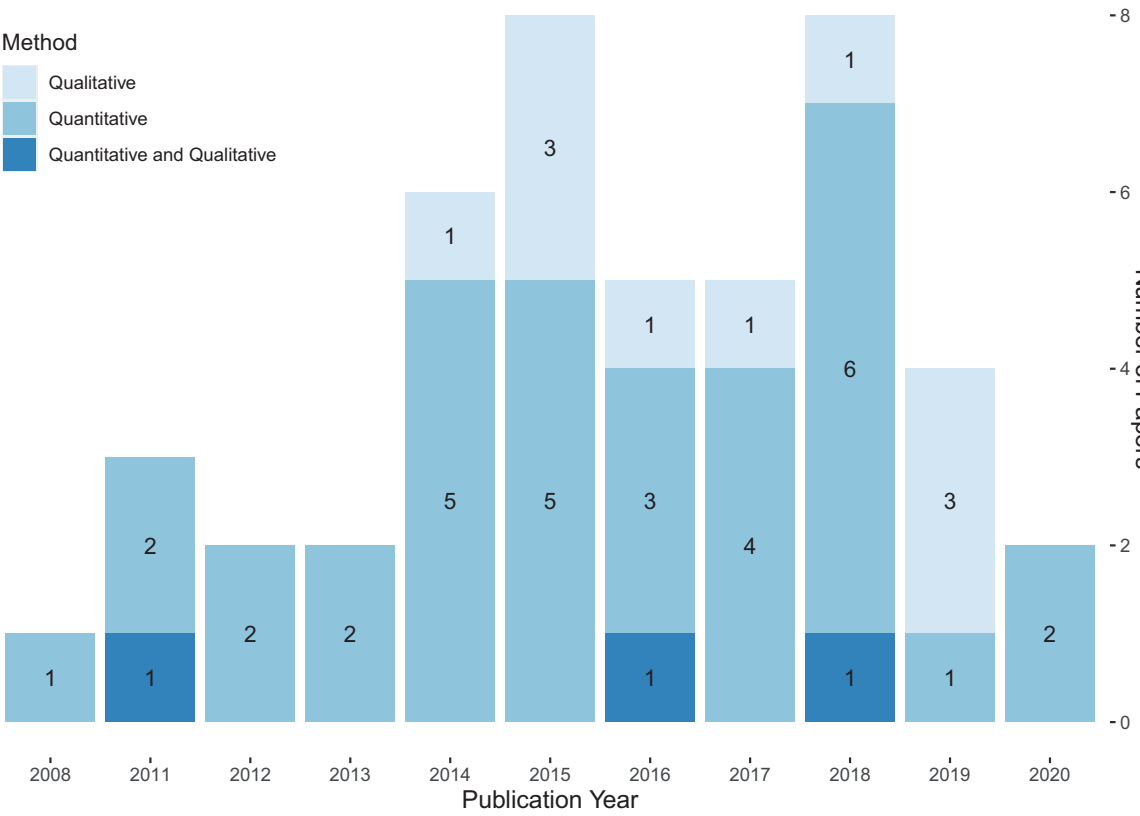

Figure 2. Literature classification by year of publication and research methods 


\section{JHOM \\ 35,9}

\section{6}

Figure 3.

Distribution of cancer types by different care services

Figure 4.

Distribution of themes found in the literature. HEA (hospital

emergency admission), DD and WTTT

(delayed diagnosis and waiting time to treatment)

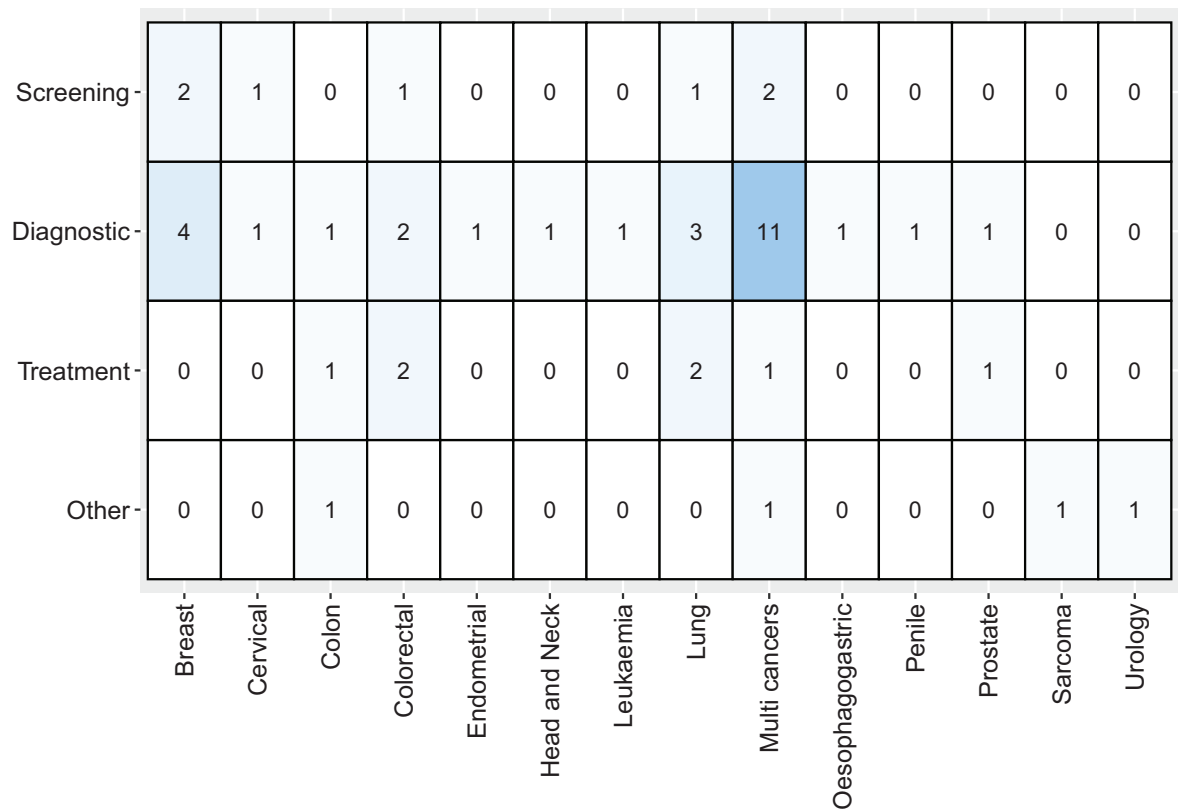

Fast Track Diagnostics

HEA

\begin{tabular}{|l|l|l|l|l|l|l|l|l|l|l|l|l|l|}
\hline 0 & 0 & 1 & 1 & 0 & 0 & 0 & 0 & 3 & 0 & 0 & 0 & 0 & 0 \\
\hline
\end{tabular}

\begin{tabular}{|l|l|l|l|l|l|l|l|l|l|l|l|l|l|}
\hline 0 & 0 & 0 & 1 & 0 & 0 & 0 & 1 & 5 & 0 & 1 & 0 & 0 & 0
\end{tabular}

Efficient Delivery

\begin{tabular}{|l|l|l|l|l|l|l|l|l|l|l|l|l|l}
2 & 0 & 0 & 2 & 0 & 1 & 0 & 0 & 3 & 0 & 0 & 1 & 1 & 1
\end{tabular}

DD and WTTT

Barriers to Care

\begin{tabular}{|c|c|c|c|c|c|c|c|c|c|c|c|c|c|}
\hline 3 & 1 & 2 & 1 & 0 & 0 & 0 & 5 & 2 & 0 & 0 & 1 & 0 & 0 \\
\hline 1 & 1 & 0 & 0 & 1 & 0 & 1 & 0 & 2 & 1 & 0 & 0 & 0 & 0 \\
\hline 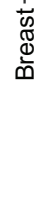 & 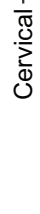 & $\frac{\overline{0}}{0}$ & $\begin{array}{l}\frac{1}{\pi} \\
\text { ป } \\
\text { d } \\
\text { 응 } \\
0\end{array}$ & 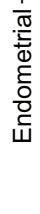 & 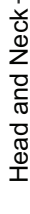 & 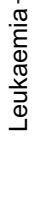 & 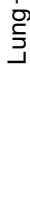 & 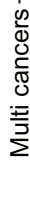 & 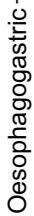 & $\begin{array}{l}\frac{\omega}{\bar{C}} \\
\frac{d}{\alpha} \\
\end{array}$ & $\begin{array}{l}\frac{0}{\pi} \\
\frac{\pi}{\omega} \\
0 \\
0\end{array}$ & 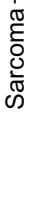 & $\begin{array}{l}\text { 웜 } \\
\text { 음 }\end{array}$ \\
\hline
\end{tabular}


intricate connections among variables, as there may be common factors that influence multiple themes. However, the discussion will be general enough to cover other parts of the diagram related to the theme.

To complement the analysis concerning individual themes, Appendix features a comprehensive picture of the system as a whole in the form of a causal loop diagram. It conveys the relationship among distinct influences of different themes, as well as the connections between the themes in terms of common influences. Even though we cannot claim that the resulting diagram is a complete picture of reality, it depicts the perceptions of the surveyed studies regarding the complex issues connected to the delivery of cancer care.

\subsection{Access barriers to care}

Figure 5 presents a causes tree highlighting factors that can compromise the access to cancer care services. Administrative barriers include poor communication and service configuration, as well as the lack of a uniform service standard for all patients (Cusimano et al., 2019). Anxiety may result from a poor relationship between doctor and patient (Clarke et al., 2014), from the natural apprehension regarding a possible cancer diagnosis (Huddy et al., 2016), or the increased tension due to a delayed diagnosis (Nessim et al., 2015).

Obesity and other co-morbidities may affect the access of patients to the point of care and produce delays in the diagnosis (Guldbrandt et al., 2015). Obesity in particular has been associated with stigma and poor communication on the part of the service provider (Cusimano et al., 2019). The cultural reluctance in seeking help contributes to delay in diagnosis and treatment, which may lead to patients presenting with late-stage cancer (Huddy et al., 2016).

Concerning financial aspects, patients from deprived areas have been found more prone to late-stage diagnosis (Maclean et al., 2015) and more likely to require emergency care (Maringe et al., 2018). Somewhat correlated are geographical factors, such as the distance to the available cancer centres (Turner et al., 2017), the area of residence of patients (Maclean et al., 2015), which is also correlated to deprivation indices (Tin et al., 2018; Tsang et al., 2013) and the availability of diagnostic procedures and treatments in the vicinity (Huddy et al., 2016). Unsurprisingly, longer travel times to cancer centres and residing in a deprived area are associated with poorer patient outcomes.

The knowledge of patients regarding the cancer types, the treatments and the awareness concerning the benefits of early treatment, play an important role in the outcomes (Redaniel et al., 2015; Momberg et al., 2017). Better informed patients tend to make better decisions regarding screening and diagnostic strategies, thereby improving early presentation indices. Finally, the understanding and trust underlying the patient-doctor relationship are also important to accelerate diagnosis and thereby improve outcomes, particularly in paediatric cancers (Clarke et al., 2014).

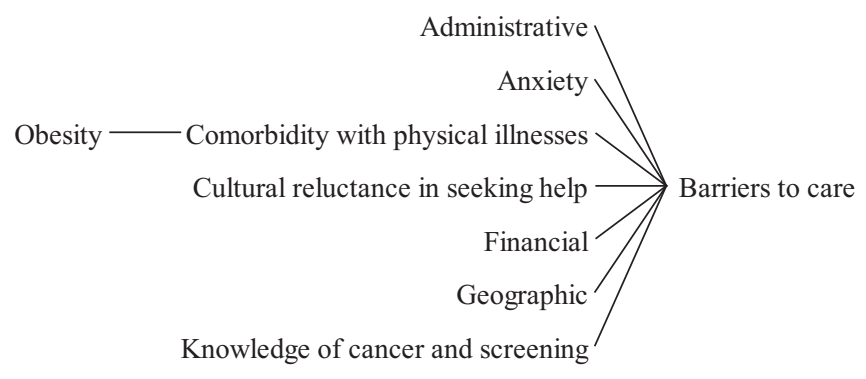

Factors influencing cancer pathways

127 . 
JHOM 35,9

128

\subsection{Efficient delivery of care pathways}

Figure 6 summarises strategies proposed and implemented to deliver improved and faster pathways. Generally, developing a cancer pathway involves prescribing performance measures and planning in accordance with these measures for post implementation and monitoring. Efficient delivery, in turn, demands leadership, coordination, information technology systems and governance (Pitter et al., 2019).

Multidisciplinary teams have a leading role in cancer care delivery. Because of this importance, multidisciplinary team meetings should be held frequently to avoid delays in diagnosis (Redaniel et al., 2015; Van Huizen et al., 2018). The literature suggests that a well-defined hierarchy can contribute to speed up treatment decisions and develop automated decisions for simple cases (Lamb et al., 2014; Redaniel et al., 2015). For rare cancer types, information technology can help identify courses of treatment when specialists are scarce (Kasper et al., 2018).

Rapid one-stop pathways have also been demonstrated effective for certain types of cancer (Bass et al., 2018; Haddow et al., 2016), and cost-effective in a more generalist setting (Sewell et al., 2020). Also essential for the delivery of a care pathway is the existence of early intervention or screening programs (Cariou et al., 2018) and the proper definition and application of standardised pathways (Hoverman et al., 2011; Kubal et al., 2016; Quan et al., 2012). This is to ensure that personalised diagnostic plans are coherent and independent of the team overseeing the pathway, and that the best experiences are shared with management and fellow specialists.

Finally, effective delivery of care requires administrative support that ensures seamless access to associated services such as psychological assistance (Franchi et al., 2013) and rehabilitation services (Stout et al., 2019). The administrative support may be a part of the cancer service which acts as a bridge between the cancer service and the associated service that is required. These supportive services may not have direct impact on the delivery of cancer pathways, nonetheless they provide support which may improve patients' experience.

\subsection{Hospital emergency admission}

Figure 7 depicts the main factors that contribute to hospital emergency admissions, according to the reviewed literature. These include age, deprivation, and comorbidity with other physical illnesses (Kreys et al., 2014; Maringe et al., 2018; Tsang et al., 2013).

Emergency visits can follow a GP referral (Black et al., 2015; Guldbrandt et al., 2015) or be completely unplanned (Ortiz-Ortiz et al., 2016). In the context of cancer pathways, unplanned emergency visits are in general a symptom of a failure in the proper delivery of care that results, for example, in late-stage diagnosis. Hence, proper prevention and screening policies

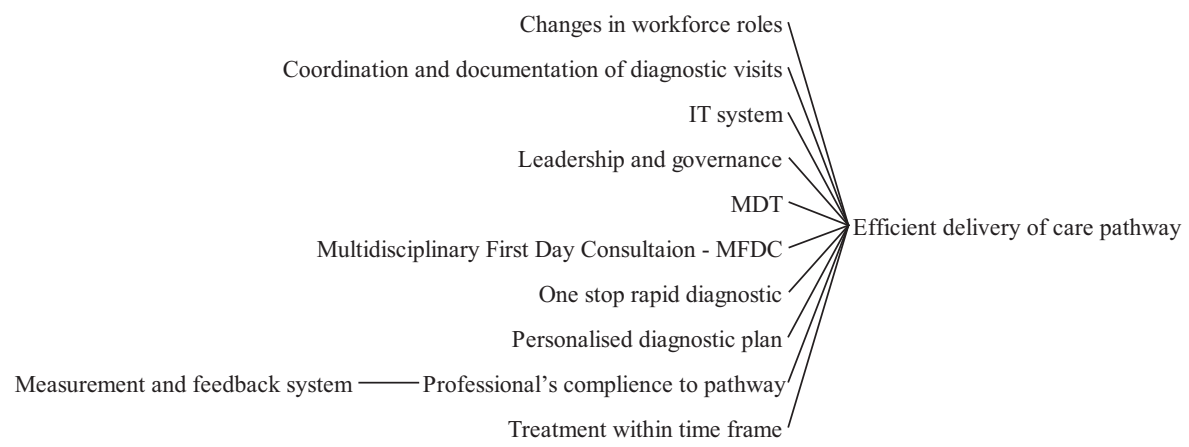

Figure 6.

Factors influencing efficiency in delivering cancer care 
(Cariou et al., 2018) as well as improved cancer awareness in primary care can help mitigate hospital emergency admissions in cancer care (Kreys et al., 2014; Tsang et al., 2013), provided that proper care is available for the patient (Turner et al., 2017).

\subsection{Fast track diagnostics}

Figure 8 summarises the factors associated with the implementation of fast track cancer pathways. Reported benefits of fast track programs include shorter diagnostic intervals and faster access to first treatment, as well as standardised protocols for a referral to secondary care (Guldbrandt et al., 2015; Jensen et al., 2014; Prades et al., 2011; Sewell et al., 2020). However, the results reported in the literature are often myopic, for they fail to consider the additional burdens imposed on non-fast-track patients. Unfortunately, the latter group is comprised of the majority of patients (Zhou et al., 2018).

Fast track services often resort to dedicated resources and prioritised use of installed capacity which intuitively lead to better delivery of care for prioritised patients (Jakobsen and Jensen, 2016; Van Harten et al., 2018). However, it is important to contrast such an improvement with the eventual degradation of the delivery of care for non-prioritised patients.

\subsection{Delay in diagnosis and waiting time to treatment}

Figure 9 highlights the contributing factors to the delay in diagnosis. As expected, there are some overlaps with the factors that lead to efficient delivery, which would be expected to prevent unnecessary delays. Figure 10 highlights the main issues that contribute to the pre-treatment and post-diagnostic delays, according to the surveyed literature.

Early diagnosis and treatment are of the utmost importance in cancer care, hence it is no surprise that time to first treatment and time to diagnosis are among the performance functions evaluated in cancer care (Black et al., 2015; Nessim et al., 2015). Delayed diagnosis can be due to administrative and systemic issues, primary care delivery, or patient related issues (Hansen et al., 2011). Patient delays are correlated to socio-demographic characteristics, such as gender, awareness of cancer, economic status, alcohol intake and tobacco consumption (Hansen et al., 2008; Huddy et al., 2016; Lim et al., 2014).

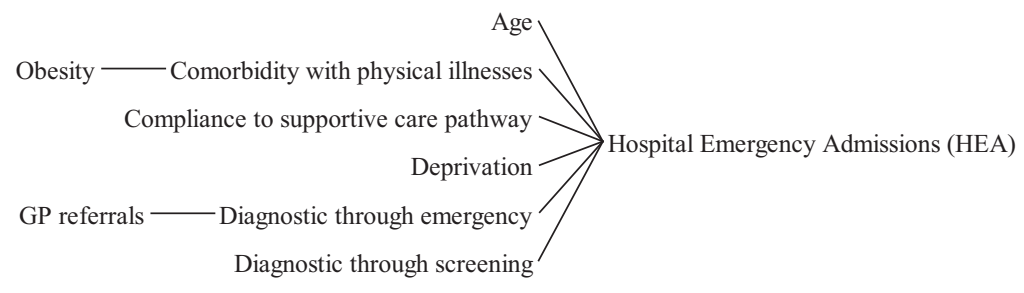

Figure 7.

Factors influencing hospital emergency admission

\footnotetext{
Close collaboration with tumor boards and diagnostic department

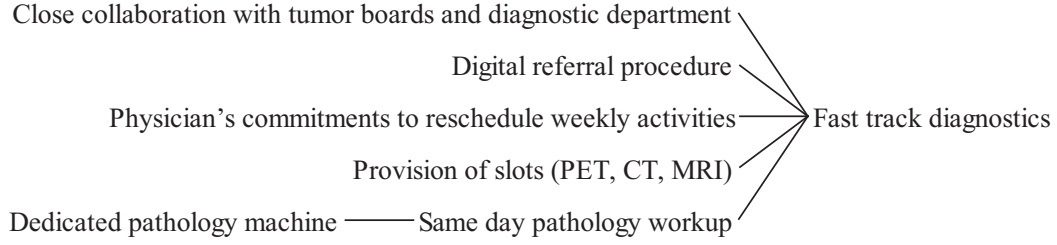

Figure 8.

Factors influencing fast track strategy 
JHOM 35,9

Figure 9.

Factors influencing delay in diagnosis
Figure 10. Factors influencing waiting time to treatment
System delay may stem from unnecessary or delayed diagnostic procedures (Chiarelli et al., 2017; Laerum et al., 2020; Héquet et al., 2017) but also from a tendency of admitting late-stage patients in the system (Forrest et al., 2014; Nessim et al., 2015; Redaniel et al., 2013). Unsurprisingly, better communication between primary and secondary care, and better qualified personnel can help mitigate system delay (Black et al., 2015). Administrative issues include mismanagement of patient transfers between services and levels of care (Iachina et al., 2017), as well as inadequate management of referral, consultation and booking for treatment (Stokstad et al., 2019; Wasserman et al., 2015).

A central nurse-led coordination of cancer care has been associated with improved outcomes and better delivery of cancer services (Aarhus et al., 2019; Blakely et al., 2015; Wulff et al., 2012). One possible reason for this is the proximity and empathy between nurses and patients (Tod et al., 2015).

\section{Discussion}

The study has identified literature pertaining to factors as well as strategies associated with the delivery of cancer care pathways from presentation to diagnosis and treatment. The analysis using qualitative mapping, in this case a causal loop diagram, revealed that factors and strategies are interlinked. It highlighted the complexity of the cancer care pathways in general, and that factors influencing a certain part of the cancer care pathway may also affect other areas in the pathway.

Access barriers to care are acknowledged in many passages of the surveyed literature, and the issues that lead to such barriers are important to both primary and secondary care. Studies that investigated access barriers sometimes have done so from the perspective of individual patients and other times have contemplated the perspective of the service providers. Factors associated with access barriers, from the patient perspective, include

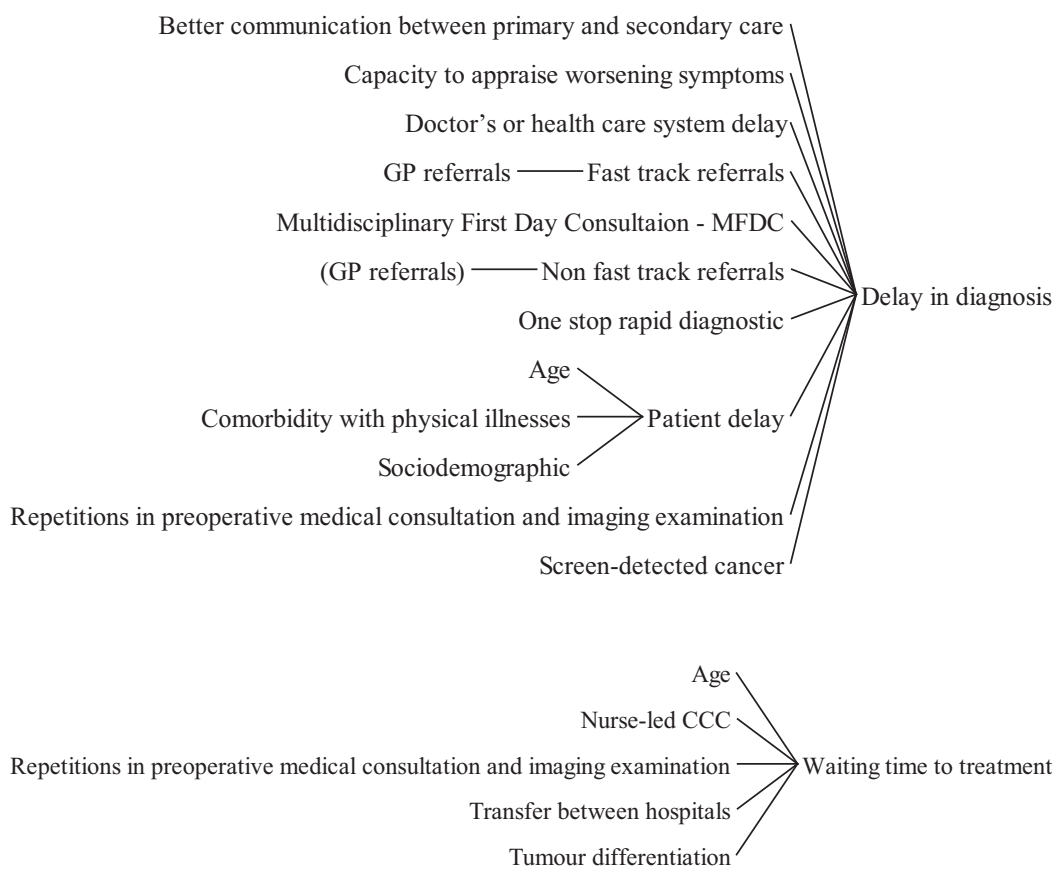


socio-economic status, demographic profile or comorbidity with other physical health conditions. These support previous reviews, such as Macleod et al. (2009) and Williams et al. (2019). The literature showed that these factors are not only associated with access to primary care but also with hospital emergency access (Tsang et al., 2013) or even hospital admissions in general.

Lack of knowledge about a certain cancer program available in the community or regarding cancer itself is a recurring theme in the literature. Such knowledge is invaluable not only for the population at risk but also for health professionals (Williams et al., 2019). It is not difficult to see that a lack of understanding about the benefits of screening programs for certain types of cancer, or about the associated diagnostic procedures, can affect the decision of individual patients on whether or not to join screening initiatives (Momberg et al., 2017). In a certain ethnicity, the lack of knowledge and awareness of cancer can be found in both developed countries (Jones et al., 2014) and developing countries (Akuoko et al., 2017).

Studies have identified a system of healthcare factors associated with the delivery of the cancer care pathway. In general, these might relate to resources such as availability of certain diagnostic procedures or treatments, the availability of experts in rare cancers, the management of diagnosis and treatment pathways, the communication between health professionals and the patients, and between service providers. These factors highlight gaps in accessing quality care in some European countries (Kasper et al., 2018). Multidisciplinary support via multidisciplinary team meetings play an important role in cancer care delivery (Lamb et al., 2014; Van Huizen et al., 2018). The effectiveness of the multidisciplinary teams depends upon the implemented strategies. These include prioritising cases based on the type and condition of the tumour and making decisions for simple cases based on a standardised cancer pathway (Lamb et al., 2014).

Patient related factors together with the healthcare system contributed to delays in diagnosis and receiving first cancer treatment (Hansen et al., 2011; Huddy et al., 2016). Raising cancer awareness in the community, and better coordination and communication between service providers are amongst suggested strategies to mitigate the delays (Black et al., 2015).

The study did not limit the delivery of care pathways for specific cancers or services. The results showed that at a high-level abstraction, factors influencing delivery at a certain phase of cancer care might be similar regardless of the cancer types. The analyses give insights into the complex care pathways, capturing not only primary care but also secondary and even tertiary care. As a result, the study presented a preliminary model toward a comprehensive description of factors and strategies influencing cancer care processes. Such a model, which provides a comprehensive knowledge regarding the cancer care pathways, may support the decision making process (Butler et al., 2013).

Finally, an apparent gap in the literature is the lack of studies analysing the holistic effects of cancer pathways in the health system (Zhou et al., 2018). In particular, the literature lacks studies evaluating the decrease in the quality of service for patients competing for the same resources when cancer care is prioritised.

\subsection{Strategies and recommendations}

Although some findings in the literature are limited to a given setting, this section exploits recurring conclusions that can be used to inform policy making in general and help optimise cancer care pathways. Firstly, it has been found that cancer awareness is positively correlated with improved outcomes and early presentation. Hence, we recommend developing policies to raise cancer awareness in the community, as well as continuous training and information exchange with healthcare professionals in primary care. Early intervention and screening plans are also very important to ensure early presentation. The intervention should be tailored to the context and address an individual's issues related to access barriers (Detterbeck et al., 2013). 
JHOM

35,9

132

Multidisciplinary teams and discussion boards should also be included in the pathways to improve outcomes and recommendations. However, these discussions should be frequent enough to prevent these meetings from becoming a bottleneck that delays diagnosis. The multidisciplinary teams should establish automated decisions for simpler cases to speed up diagnosis. This may be supported by extending the role of the cancer nurse specialists in the multidisciplinary teams.

Pathways should be standardised to ensure that diagnostic plans are independent of the team overseeing the pathway, and information exchange should guarantee that the best experiences are shared with management and fellow specialists. The pathways should provide a comprehensive cancer care program, be implemented, and subject to regular update (Christensen et al., 2017). In addition, proper information technology (IT) support and rapid access to associated services such as psychological assistance and rehabilitation services should be ensured to improve outcomes.

Finally, fast track diagnostics should be considered to speed up treatment decisions. However, the planning of a fast track service should consider the impact to all patients that make use of the shared resource that would be prioritised, to make sure that the overall effect is positive. To avoid delays, preemptive measures should also be considered to prevent delays related to socio-economic and demographic characteristics. Finally, a channel should be established between primary and secondary care, as such a channel has been associated with delay mitigation (Brown et al., 2014).

\subsection{Study limitation}

Due to the keywords and inclusion criteria used for the literature search, the study may not have covered the literature in its entirety. The search was limited to articles published in journals. Future studies may update this effort and include grey literature such as policy and organisational reports.

The resultant causal loop diagram model was developed using combined factors and strategies discussed in the included studies. In addition, the quality of each study was not assessed and has not been taken into account in the analysis. Hence, the resulting diagram might capture the subjectivity of the authors in summarizing the results, as well as biases found in the studies. Further research might contest and refine the model by including more evidence from studies. Others might take some ideas presented in the model and turn them into a quantitative model that can be used to investigate the interrelationship between the factors. Such a quantitative model might capture not only the patient flow in the cancer care system but also the factors influencing the flow.

The study is limited to cancer care pathways in relation to diagnostic and treatment delivery. Factors related to patient outcomes such as survival and quality of life were not included. Future studies may include literature discussing patient outcomes in relation to cancer care pathways. The inclusion of such literature may highlight the important links between other support care pathways and the diagnostic and treatment care pathways. This would be a step towards capturing a holistic view of healthcare systems in cancer care.

\section{Conclusion}

Factors influencing the delivery of cancer pathways are myriad and complex. In general, the factors may relate to the individual patient or the system of care. Factors such as patient characteristics, socio-economic conditions, knowledge of cancer and cancer symptoms are interrelated and influence different cancer services. The results not only highlighted the factors associated with delay in diagnosis or treatment, but also the strategies proposed in the literature to deliver timely cancer pathways, such as fast track diagnostics. 
The successful delivery of cancer pathways was supported by factors such as IT and information systems, multidisciplinary teams, and case management, among others. However, the number of studies found is not large, especially with respect to specific cancer types. More studies are needed on the successful delivery of cancer pathways, particularly focusing on specific cancer types, as different cancers require distinct cancer pathways.

This study provided, by means of a causal loop diagram, a comprehensive picture of the factors influencing cancer care. The resulting model developed in the study can be regarded as a preliminary model representing complex cancer care pathways. Future studies should confirm or mitigate the links between factors by including up-to-date evidence. The findings give rise to recommendations and insights that can guide practitioners in the development of new and the refinement of existing cancer care pathways.

\section{References}

Aarhus, R., Tjørnhøj-Thomsen, T., Tarp, B., Vedsted, P. and Andersen, R.S. (2019), "Coordinating objects of care: exploring the role of case managers as brokers in cancer patient pathways", European Journal of Cancer Care, Vol. 28 No. 3, p. e13017, doi: 10.1111/ecc.13017.

Akuoko, C., Armah, E., Sarpong, T., Quansah, D., Amankwaa, I. and Boateng, D. (2017), "Barriers to early presentation and diagnosis of breast cancer among African women living in sub-Saharan Africa”, PLoS One, Vol. 12 No. 2, p. e0171024, doi: 10.1371/journal.pone.0171024.

Allen, D., Purkis, M.E., Rafferty, A.M. and Obstfelder, A. (2019), "Integrating preparation for care trajectory management into nurse education: competencies and pedagogical strategies", Nursing Inquiry, Vol. 26 No. 3, p. e12289, doi: 10.1111/nin.12289.

Altini, M., Solinas, L., Bucchi, L., Gentili, N., Gallegati, D., Balzi, W., Falcini, F. and Massa, I. (2020), "Assessment of cancer care costs in disease-specific cancer care pathways", International Journal of Environmental Research and Public Health, Vol. 17 No. 13, p. 4765, doi: 10.3390/ ijerph17134765.

Alzahouri, K., Velten, M., Arveux, P., Woronoff-Lemsi, M.C., Jolly, D. and Guillemin, F. (2008), "Management of SPN in France. Pathways for definitive diagnosis of solitary pulmonary nodule: a multicentre study in 18 French districts”, BMC Cancer, Vol. 8 No. 1, p. 93, doi: 10.1186/ 1471-2407-8-93.

Bass, E., Freeman, A., Jameson, C., Punwani, S., Moore, C., Arya, M., Emberton, M. and Ahmed, H. (2018), "Prostate cancer diagnostic pathway: is a one-stop cognitive MRI targeted biopsy service a realistic goal in everyday practice? A pilot cohort in a tertiary referral centre in the UK”, BMJ Open, Vol. 8 No. 10, p. e024941, doi: 10.1136/bmjopen-2018-024941.

Black, G., Sheringham, J., Spencer-Hughes, V., Ridge, M., Lyons, M., Williams, C., Fulop, N. and Pritchard-Jones, K. (2015), "Patients' experiences of cancer diagnosis as a result of an emergency presentation: a qualitative study", PLoS One, Vol. 10 No. 8, p. e0135027, doi: 10.1371/ journal.pone.0135027.

Blakely, T., Collinson, L., Kvizhinadze, G., Nair, N., Foster, R., Dennett, E. and Sarfati, D. (2015), "Cancer care coordinators in stage III colon cancer: a cost-utility analysis", BMC Health Services Research, Vol. 15 No. 306, doi: 10.1186/s12913-015-0970-5.

Bosscher, M., Van Leeuwen, B. and Hoekstra, H. (2015), "Current management of surgical oncologic emergencies”, PLoS One, Vol. 10 No. 5, p. e0124641, doi: 10.1371/journal.pone.0124641.

Brown, S., Castelli, M., Hunter, D.J., Erskine, J., Vedsted, P., Foot, C. and Rubin, G. (2014), "How might healthcare systems influence speed of cancer diagnosis: a narrative review", Social Science and Medicine, Vol. 116 No. 100, pp. 56-63, doi: 10.1016/j.socscimed.2014.06.030.

Butler, J., Foot, C., Bomb, M., Hiom, S., Coleman, M., Bryant, H., Vedsted, P., Hanson, J. and Richards, M. and ICBP Working Group (2013), "The international cancer benchmarking partnership: an international collaboration to inform cancer policy in Australia, Canada, Denmark, Norway, 
JHOM 35,9
Sweden and the United Kingdom”, Health Policy, Vol. 112 Nos 1-2, pp. 148-155, doi: 10.1016/j. healthpol.2013.03.021.

Cancer Care Ontario (2019), "Lung cancer diagnosis pathway map", Ontario Health, available at: https://www.cancercareontario.ca/en/pathway-maps/lung-cancer (accessed 7 May 2019).

Cariou, A., Rouzier, R., Baffert, S., Soilly, A.L. and Héquet, D. (2018), "Multidimensional impact of breast cancer screening: results of the multicenter prospective optisoins01 study", PLoS One, Vol. 13 No. 8, p. e0202385, doi: 10.1371/journal.pone.0202385.

Chiarelli, A., Muradali, D., Blackmore, K., Smith, C., Mirea, L., Majpruz, V., O’Malley, F., Quan, M. and Holloway, C. (2017), "Evaluating wait times from screening to breast cancer diagnosis among women undergoing organised assessment vs usual care", British Journal of Cancer, Vol. 116 No. 10, pp. 1254-1263, doi: 10.1038/bjc.2017.87.

Christensen, N.L., Jekunen, A., Heinonen, S., Dalton, S.O. and Rasmussen, T.R. (2017), "Lung cancer guidelines in Sweden, Denmark, Norway and Finland: a comparison”, Acta Oncologica, Vol. 56 No. 7, pp. 943-948, doi: 10.1080/0284186X.2017.1315172.

Clarke, R., Jones, C., Mitchell, C. and Thompson, M. (2014), "Shouting from the roof top: a qualitative study of how children with leukaemia are diagnosed in primary care", BMJ Open, Vol. 4 No. 2, p. e004640, doi: 10.1136/bmjopen-2013-004640.

Currie, V.L. and Harvey, G. (2000), "The use of care pathways as tools to support the implementation of evidence-based practice", Journal of Interprofessional Care, Vol. 14 No. 4, pp. 311-324, doi: 10. 1080/13561820020003874.

Cusimano, M.C., Simpson, A.N., Han, A., Hayeems, R., Bernardini, M.Q., Robertson, D., Kives, S.L., Satkunaratnam, A., Baxter, N.N. and Ferguson, S.E. (2019), "Barriers to care for women with low-grade endometrial cancer and morbid obesity: a qualitative study", BMJ Open, Vol. 9 No. 6, p. e026872, doi: 10.1136/bmjopen-2018-026872.

Delilovic, S., Hasson, H., Åhström, M. and von Knorring, M. (2019), "Implementing standardized cancer patient pathways (CPPs) - a qualitative study exploring the perspectives of health care professionals”, BMC Health Services Research, Vol. 19 No. 577, doi: 10.1186/s12913-019-4413-6.

Department of Health (2007), Cancer Reform Strategy, Department of Health, London, available at: https://webarchive.nationalarchives.gov.uk/20130104165259/http://www.dh.gov.uk/en/ Publicationsandstatistics/Publications/PublicationsPolicyAndGuidance/DH_081006 (accessed 30 September 2020).

Department of Health and Human Services (2016), Optimal Care Pathway for People with Lung Cancer, Cancer Australia and Cancer Council, available at: https://www.cancer.org.au/content/ocp/ health/optimal-care-pathway-for-people-with-lung-cancer-june-2016.pdf (accessed 07 May 2019).

Detterbeck, F.C., Mazzone, P.J., Naidich, D.P. and Bach, P.B. (2013), "Screening for lung cancer: diagnosis and management of lung cancer: American College of Chest Physicians evidencebased clinical practice guidelines”, Chest, Vol. 143 No. 5, pp. e78S-e92S, doi: 10.1378/chest. $12-2350$.

Donetto, S., Desai, A., Zoccatelli, G., Robert, G., Allen, D., Brearly, S. and Raffetty, A.M. (2019), "Organisational strategies and practices to improve care using patient experience data in acute NHS hospital trusts: an ethnographic study", Health Services and Delivery Research, NIHR Journals Library, Southampton (UK), Vol. 34 No. 7, doi: 10.3310/hsdr07340.

Forrest, L.F., Adams, J., White, M. and Rubin, G. (2014), "Factors associated with timeliness of postprimary care referral, diagnosis and treatment for lung cancer: population-based, data-linkage study", British Journal of Cancer, Vol. 111 No. 9, pp. 1843-1851, doi: 10.1038/bjc.2014.472.

Franchi, G., Bulli, F., Muraca, M., Maruelli, A., Grechi, E. and Miccinesi, G. (2013), "Impact of a psychooncological rehabilitation intervention on psychological distress: the experience of CeRiON (Oncological Rehabilitation Centre) Florence 2007-2010", Supportive Care in Cancer, Vol. 21 No. 9, pp. 2381-2386, doi: 10.1007/s00520-013-1803-x.

Gesme, B. and Wiseman, M. (2011), "Strategic use of clinical pathways", Journal of Oncology Practice, Vol. 7 No. 1, pp. 54-56, doi: 10.1200/JOP.2010.000193. 
Guldbrandt, L., Fenger-Grøn, M., Rasmussen, T., Jensen, H. and Vedsted, P. (2015), “The role of general practice in routes to diagnosis of lung cancer in Denmark: a population-based study of general practice involvement, diagnostic activity and diagnostic intervals", BMC Health Services Research, Vol. 15 No. 21, doi: 10.1186/s12913-014-0656-4.

Haddow, J.B., Walshe, M., Aggarwal, D., Thapar, A., Hardman, J., Wilson, J., Oshowo, A., Bhan, C. and Mukhtar, H. (2016), "Improving the diagnostic stage of the suspected colorectal cancer pathway: a quality improvement project”, Healthcare, Vol. 4 No. 3, pp. 225-234, doi: 10.1016/j.hjdsi.2015. 09.004 .

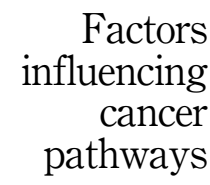

135

Hansen, R., Olesen, F., Sørensen, H., Sokolowski, I. and Søndergaard, J. (2008), "Socioeconomic patient characteristics predict delay in cancer diagnosis: a Danish cohort study", BMC Health Services Research, Vol. 8 No. 49, doi: 10.1186/1472-6963-8-49.

Hansen, R., Vedsted, P., Sokolowski, I., Søndergaard, J. and Olesen, F. (2011), "Time intervals from first symptom to treatment of cancer: a cohort study of 2,212 newly diagnosed cancer patients", BMC Health Services Research, Vol. 11 No. 284, doi: 10.1186/1472-6963-11-284.

Héquet, D., Huchon, C., Baffert, S., Alran, S., Reyal, F., Nguyen, T., Combes, A., Trichot, C., Alves, K., Berseneff, H. and Rouzier, R. (2017), "Preoperative clinical pathway of breast cancer patients: determinants of compliance with EUSOMA quality indicators", British Journal of Cancer, Vol. 116 No. 11, pp. 1394-1401, doi: 10.1038/bjc.2017.114.

Hoverman, J.R., Cartwright, T.H., Patt, D.A., Espirito, J.L., Clayton, M.P., Garey, J.S., Kopp, T.J., Kolodziej, M., Neubauer, M., Fitch, K., Pyenson, B. and Beveridge, R. (2011), "Pathways, outcomes, and costs in colon cancer: retrospective evaluations in two distinct databases", Journal of Oncology Practice, Vol. 7 No. 3S, pp. 52s-59s, doi: 10.1200/JOP.2011.000318.

Huddy, J., Weldon, S.M., Ralhan, S., Painter, T., Hanna, G., Kneebone, R. and Bello, F. (2016), "Sequential simulation (SQS) of clinical pathways: a tool for public and patient engagement in point-of-care diagnostics”, BMJ Open, Vol. 6, p. e011043, doi: 10.1136/bmjopen-2016-011043.

Iachina, M., Jakobsen, E., Fallesen, A. and Green, A. (2017), "Transfer between hospitals as a predictor of delay in diagnosis and treatment of patients with non-small cell lung cancer - a register based cohort-study", BMC Health Services Research, Vol. 17 No. 267, doi: 10.1186/s12913-0172230-3.

Jakobsen, J.K. and Jensen, J.B. (2016), "DaPeCa-2: implementation of fast-track clinical pathways for penile cancer shortens waiting time and accelerates the diagnostic process-a comparative before-and-after study in a tertiary referral centre in Denmark", Scandinavian Journal of Urology, Vol. 50 No. 1, pp. 80-87, doi: 10.3109/21681805.2015.1077472.

Jensen, H., Tørring, M.L., Olesen, F., Overgaard, J. and Vedsted, P. (2014), "Cancer suspicion in general practice, urgent referral and time to diagnosis: a population-based GP survey and registry study”, BMC Cancer, Vol. 14 No. 636, doi: 10.1186/1471-2407-14-636.

Jensen, H., Tørring, M.L. and Vedsted, P. (2017), "Prognostic consequences of implementing cancer patient pathways in Denmark: a comparative cohort study of symptomatic cancer patients in primary care”, BMC Cancer, Vol. 17 No. 627, doi: 10.1186/s12885-017-3623-8.

Jones, C., Maben, J., Jack, R., Davies, E., Forbes, L., Lucas, G. and Ream, E. (2014), “A systematic review of barriers to early presentation and diagnosis with breast cancer among Black women", BMJ Open, Vol. 4 No. 2, p. e004076, doi: 10.1136/bmjopen-2013-004076.

Kasper, B., Lecointe-Artzner, E., Wait, S., Boldon, S., Wilson, R., Gronchi, A., Valverde, C., Eriksson, M., Dumont, S., Drove, N., Kanli, A. and Wartenberg, M. (2018), "Working to improve the management of sarcoma patients across Europe: a policy checklist", BMC Cancer, Vol. 18 No. 424, doi: 10.1186/s12885-018-4320-y.

Kreys, E., Kim, T., Delgado, A. and Koeller, J. (2014), "Impact of cancer supportive care pathways compliance on emergency department visits and hospitalizations", Journal of Oncology Practice, Vol. 10 No. 3, pp. 168-173, doi: 10.1200/JOP.2014.001376.

Kubal, T., Letson, D., Chiappori, A., Springett, G., Shimkhada, R., Tamondong Lachica, D. and Peabody, J. (2016), "Longitudinal cohort study to determine effectiveness of a novel simulated 
JHOM 35,9

case and feedback system to improve clinical pathway adherence in breast, lung and GI cancers", BMJ Open, Vol. 6 No. 9, p. e012312, doi: 10.1136/bmjopen-2016-012312.

Laerum, D., Brustugun, O.T., Gallefoss, F., Falk, R., Strand, T.E. and Fjellbirkeland, L. (2020), "Reduced delays in diagnostic pathways for non-small cell lung cancer after local and National initiatives", Cancer Treatment and Research Communications, Vol. 23, p. e100168, doi: 10.1016/j. ctarc.2020.100168.

Lamb, B.W., Jalil, R.T., Sevdalis, N., Vincent, C. and Green, J.S.A. (2014), "Strategies to improve the efficiency and utility of multidisciplinary team meetings in urology cancer care: a survey study", BMC Health Services Research, Vol. 14 No. 377, doi: 10.1186/1472-6963-14-377.

Lawson, D., Revelino, K. and Owen, D. (2006), "Clinical pathways to improve patient outcomes", Physical Therapy Reviews, Vol. 11 No. 4, pp. 269-272, doi: 10.1179/108331906X144118.

Lim, A.W., Ramirez, A.J., Hamilton, W., Sasieni, P., Patnick, J. and Forbes, L.J. (2014), "Delays in diagnosis of young females with symptomatic cervical cancer in England: an interview-based study", British Journal of General Practice, Vol. 64 No. 627, pp. e602-e610, doi: 10.3399/bjgp14X681757.

Littlejohns, L.B., Baum, F., Lawless, A. and Freman, T. (2018), "The value of a causal loop diagram in exploring the complex interplay of factors that influence health promotion in a multisectoral health system in Australia”, Health Research Policy and Systems, Vol. 16 No. 126, doi: 10.1186/ s12961-018-0394-x.

Lung Clinical Expert Group (2017), "National optimal lung cancer pathway", available at: https:// www.cancerresearchuk.org/sites/default/files/national_optimal_lung_pathway_aug_2017.pdf (accessed 5 April 2019).

Maclean, R., Jeffreys, M., Ives, A., Jones, T., Verne, J. and Ben-Shlomo, Y. (2015), "Primary care characteristics and stage of cancer at diagnosis using data from the National Cancer Registration Service, quality outcomes framework and general practice information”, $B M C$ Cancer, Vol. 15 No. 500, doi: 10.1186/s12885-015-1497-1.

Macleod, U., Mitchell, E., Burgess, C., Macdonald, S. and Ramirez, A. (2009), "Risk factors for delayed presentation and referral of symptomatic cancer: evidence for common cancers", British Journal of Cancer, Vol. 101, pp. S92-S101, doi: 10.1038/sj.bjc.6605398.

Maringe, C., Rachet, B., Lyratzopoulos, G. and Rubio, F. (2018), "Persistent inequalities in unplanned hospitalisation among colon cancer patients across critical phases of their care pathway, England, 2011-13”, British Journal of Cancer, Vol. 119 No. 5, pp. 551-557, doi: 10.1038/s41416018-0170-2.

Momberg, M., Botha, M., Van Der Merwe, F. and Moodley, J. (2017), "Women’s experiences with cervical cancer screening in a colposcopy referral clinic in Cape Town, South Africa: a qualitative analysis", BMJ Open, Vol. 7, p. e013914, doi: 10.1136/bmjopen-2016-013914.

Nessim, C., Winocour, J., Holloway, D., Saskin, R. and Holloway, C. (2015), "Wait times for breast cancer surgery: effect of magnetic resonance imaging and preoperative investigations on the diagnostic pathway", Journal of Oncology Practice, Vol. 11 No. 2, pp. e131-e138, doi: 10.1200/JOP. 2014.002105 .

Ortiz-Ortiz, K., Ros-Motta, R., Marn-Centeno, H., Cruz-Correa, M. and Ortiz, A. (2016), "Factors associated with late stage at diagnosis among Puerto Rico's government health plan colorectal cancer patients: a cross-sectional study”, BMC Health Services Research, Vol. 16 No. 344, doi: 10.1186/s12913-016-1590-4.

Pitter, J., Csanádi, M., Szigeti, A., Lukács, G., Kovács, Á., Moizs, M., Repa, I., Zemplényi, A., Czypionka, T., Kraus, M., Rutten-Van Mölken, M. and Kaló, Z. (2019), "Planning, implementation and operation of a personalized patient management system for subjects with first suspect of cancer (Onkonetwork): system description based on a qualitative study", BMC Health Services Research, Vol. 19 No. 131, doi: 10.1186/s12913-019-3957-9.

Prades, J., Espinàs, J., Font, R., Argimon, J. and Borràs, J. (2011), "Implementing a Cancer Fast-track Programme between primary and specialised care in Catalonia (Spain): a mixed methods study", British Journal of Cancer, Vol. 105 No. 6, pp. 753-759, doi: 10.1038/bjc.2011.308. 
Prager, G.W., Braga, S., Bystricky, B., Qvortrup, C., Criscitiello, C., Esin, E., Sonke, G.S., Martínez, G.A., Frenel, J., Karamouzis, M., Strijbos, M., Yazici, O., Bossi, P., Banerjee, S., Troiani, T., Eniu, A., Ciardiello, F., Tabernero, J.Z., Zielinski, C.C., Casali, P.G., Cardoso, F., Douillard, J., Jezdic, S., McGregor, K., Bricalli, G., Vyas, M. and Ilbawi, A. (2018), "Global cancer control: responding to the growing burden, rising costs and inequalities in access", ESMO Open, Vol. 3 No. 2, p. e000285, doi: 10.1136/esmoopen-2017-000285.

Quan, M., Shumak, R., Majpruz, V., Holloway, C., O’Malley, F. and Chiarelli, A. (2012), "Improving work-up of the abnormal mammogram through organized assessment: results from the Ontario breast screening program", Journal of Oncology Practice, Vol. 8 No. 2, pp. 107-112, doi: 10.1200/ JOP.2011.000413.

Queen Mary University of London (2017), "New research reveals one in two people in the UK will get cancer", available at: https://www.qmul.ac.uk/wolfson/about-us/news/items/new-researchreveals-one-in-two-people-in-the-uk-will-get-cancer.html (accessed 12 January 2021).

Redaniel, M., Martin, R., Gillatt, D., Wade, J. and Jeffreys, M. (2013), "Time from diagnosis to surgery and prostate cancer survival: a retrospective cohort study", BMC Cancer, Vol. 13 No. 559, doi: 10.1186/1471-2407-13-559.

Redaniel, M., Ridd, M., Martin, R., Coxon, F., Jeffreys, M. and Wade, J. (2015), "Rapid diagnostic pathways for suspected colorectal cancer: views of primary and secondary care clinicians on challenges and their potential solutions", BMJ Open, Vol. 5 No. 10, p. e008577, doi: 10.1136/ bmjopen-2015-008577.

Saghatchian, M., Thonon, F., Boomsma, F., Hummel, H., Koot, B., Harrison, C., Rajan, A., De Valeriola, D., Otter, R., Pontes, J., Lombardo, C., McGrath, E., Ringborg, U., Tursz, T. and Van Harten, W. (2014), "Pioneering quality assessment in European cancer centers: a data analysis of the organization for European cancer institutes accreditation and designation program", Journal of Oncology Practice, Vol. 10 No. 5, pp. e342-e349, doi: 10.1200/JOP.2013.001331.

Schrijvers, G., van Hoorn, A. and Huiskes, N. (2012), "The care pathway: concepts and theories: an introduction”, International Journal of Integrated Care, Vol. 12, p. e192, Spec Ed Integrated Care Pathways, doi: 10.5334/ijic.812.

Sewell, B., Jones, M., Gray, H., Wilkes, H., Lloyd-Bennett, C., Beddow, K., Bevan, M. and Fitzsimmons, D. (2020), "Rapid cancer diagnosis for patients with vague symptoms: a cost-effectiveness study", British Journal of General Practice, Vol. 70 No. 692, pp. e186-e192, doi: 10.3399/bjgp20X708077.

Sterman, J. (2000), Business Dynamics: Systems Thinking and Modeling for a Complex World, Irwin/ McGraw-Hill, Boston.

Stokstad, T., Sørhaug, S., Amundsen, T. and Grønberg, B.H. (2019), "Reasons for prolonged time for diagnostic workup for stage I-II lung cancer and estimated effect of applying an optimized pathway for diagnostic procedures", BMC Health Services Research, Vol. 19 No. 679, pp. 1-9, doi: 10.1186/s12913-019-4517-z.

Stout, N., Sleight, A., Pfeiffer, D., Galantino, M. and deSouza, B. (2019), "Promoting assessment and management of function through navigation: opportunities to bridge oncology and rehabilitation systems of care", Supportive Care in Cancer, Vol. 27 No. 12, pp. 4497-4505, doi: 10.1007/s00520-019-04741-0.

Tin Tin, S., Elwood, J., Brown, C., Sarfati, D., Campbell, I., Scott, N., Ramsaroop, R., Seneviratne, S., Harvey, V. and Lawrenson, R. (2018), "Ethnic disparities in breast cancer survival in New Zealand: which factors contribute?", BMC Cancer, Vol. 18 No. 1, p. 58, doi: 10.1186/s12885-017-3797-0.

Tod, A., Redman, J., McDonnel, A., Borthwick, D. and White, J. (2015), "Lung cancer treatment rates and the role of the lung cancer nurse specialist: a qualitative study", BMJ Open, Vol. 5 No. 12, p. e008587, doi: 10.1136/bmjopen-2015-008587.

Tsang, C., Bottle, A., Majeed, A. and Aylin, P. (2013), "Cancer diagnosed by emergency admission in England: an observational study using the general practice research database", BMC Health Services Research, Vol. 13 No. 308, doi: 10.1186/1472-6963-13-308. 
JHOM 35,9
Turner, M., Fielding, S., Ong, Y., Dibben, C., Feng, Z., Brewster, D., Black, C., Lee, A. and Murchie, P. (2017), "A cancer geography paradox? Poorer cancer outcomes with longer travelling times to healthcare facilities despite prompter diagnosis and treatment: a data-linkage study", British Journal of Cancer, Vol. 117 No. 3, pp. 439-449, doi: 10.1038/bjc.2017.180.

Van Harten, W., Goedbloed, N., Boekhout, A. and Heintzbergen, S. (2018), "Implementing large scale fast track diagnostics in a comprehensive cancer center, pre- and post-measurement data", BMC Health Services Research, Vol. 18 No. 85, doi: 10.1186/s12913-018-2868-5.

Van Huizen, L., Dijkstra, P., Van Der Laan, B., Reintsema, H., Ahaus, K., Bijl, H. and Roodenburg, J. (2018), "Multidisciplinary first-day consultation accelerates diagnostic procedures and throughput times of patients in a head-and-neck cancer care pathway, a mixed method study", BMC Health Services Research, Vol. 18 No. 820, doi: 10.1186/s12913-018-3637-1.

Wang, F. and Onega, T. (2015), “Accessibility of cancer care: disparities, outcomes and mitigation”, Annals of GIS, Vol. 21 No. 2, pp. 119-125, doi: 10.1080/19475683.2015.1007893.

Wasserman, D., Boulos, M., Hopman, W., Booth, C., Goodwin, R. and Biagi, J. (2015), "Reasons for delay in time to initiation of adjuvant chemotherapy for colon cancer", Journal of Oncology Practice, Vol. 11 No. 1, pp. e28-e35, doi: 10.1200/JOP.2014.001531.

Weller, D., Vedsted, P., Rubin, G., Walter, F., Emery, J., Scott, S., Campbell, C., Andersen, R.S., Hamilton, W., Olesen, F., Rose, P., Nafees, S., van Rijswijk, E., Hiom, S., Muth, C., Beyer, M. and Neal, R.D. (2012), "The Aarhus statement: improving design and reporting of studies on early cancer diagnosis”, British Journal of Cancer, Vol. 106 No. 7, pp. 1262-1267, doi: 10.1038/bjc. 2012.68.

Williams, P., Murchie, P. and Bond, C. (2019), "Patient and primary care delays in the diagnostic pathway of gynaecological cancers: a systematic review of influencing factors", British Journal of General Practice, Vol. 69 No. 679, pp. e106-e111, doi: 10.3399/bjgp19X700781.

World Health Organization (2018), "Cancer", available at: https://www.who.int/news-room/fact-sheets/ detail/cancer (accessed 01 May 2019).

Wulff, C.N., Thygesen, M., Søndergaard, J. and Vedsted, P. (2008), "Case management used to optimize cancer care pathways: a systematic review", BMC Health Services Research, Vol. 8 No. 227, doi: 10.1186/1472-6963-8-227.

Wulff, C.N., Vedsted, P. and Søndergaard, J. (2012), “A randomised controlled trial of hospital-based case management to improve colorectal cancer patients' health-related quality of life and evaluations of care", BMJ Open, Vol. 2 No. 6, p. e001481, doi: 10.1136/bmjopen-2012-001481.

Zhou, Y., Mendonca, S., Abel, G., Hamilton, W., Walter, F., Johnson, S., Shelton, J., Elliss-Brookes, L., McPhail, S. and Lyratzopoulos, G. (2018), "Variation in 'fast-track' referrals for suspected cancer by patient characteristic and cancer diagnosis: evidence from 670000 patients with cancers of 35 different sites”, British Journal of Cancer, Vol. 118 No. 1, pp. 24-31, doi: 10.1038/bjc.2017.381.

\section{Corresponding author}

Syaribah Noor Brice can be contacted at: BriceSN@Cardiff.ac.uk 


\section{Appendix}

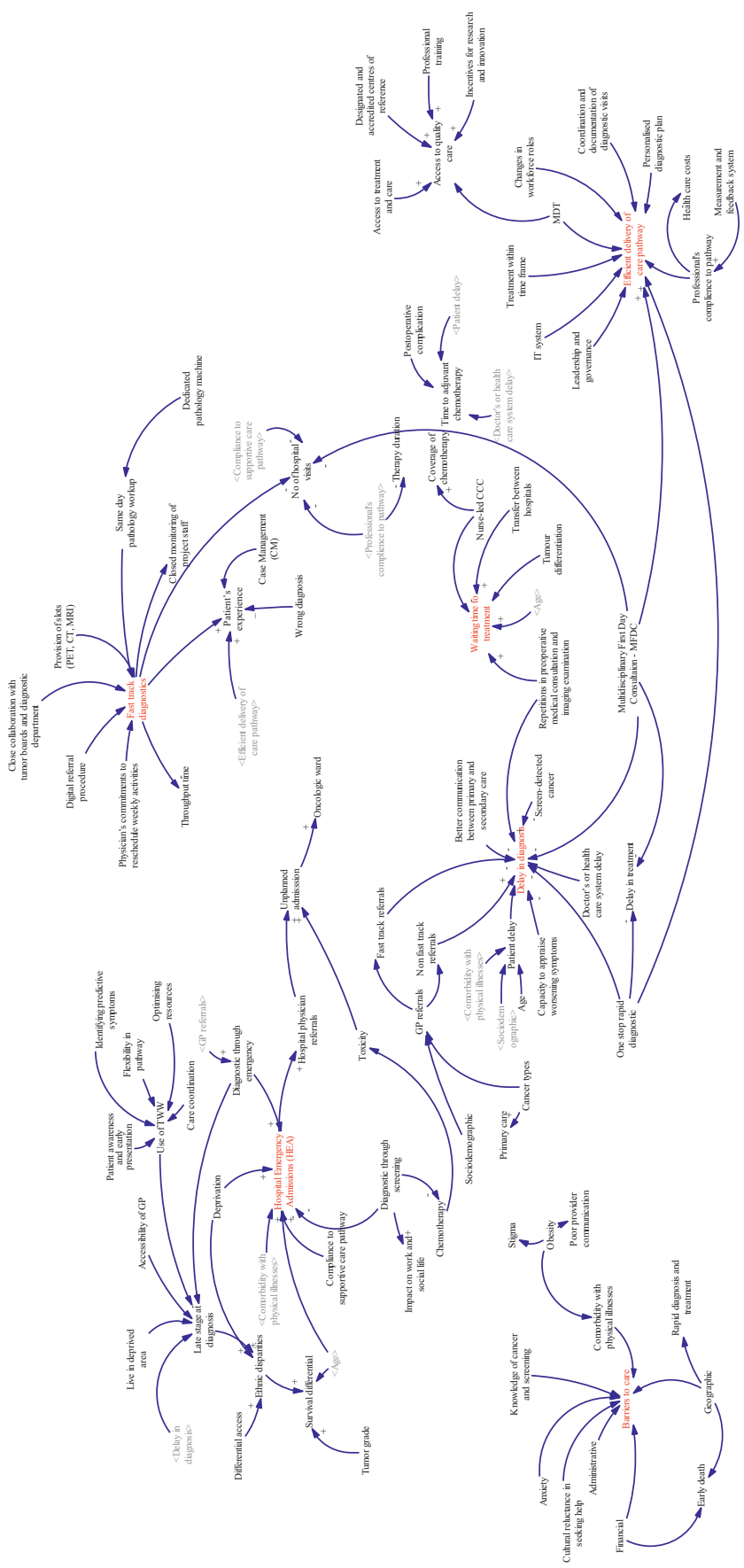

Factors influencing cancer pathways

139
Figure A1. CLD Diagram for factors influencing the delivery of cancer pathways, developed using Vensim PLE 